\title{
Effect of crystalline disorder on magnetic switching in small magnetic cells
}

\author{
Daniel Braun* \\ Infineon Technologies, 2070 State Route 52, Hopewell Junction, NY, 12533 \\ MRAM Developement Alliance, IBM/Infineon Technologies, IBM Semiconductor Research and \\ Developement Center, 2070 State Route 52, Hopewell Junction, NY, 12533
}

November 8, 2018

\begin{abstract}
I present a study of the influence of disorder in thin magnetic films on the switching behavior of small magnetic cells of well defined shape and size. The disorder considered arises from randomly oriented crystalline grains of different shape, size, and crystalline orientation which gives rise to locally fluctuating intrinsic anisotropy directions and strengths. The study comprises a theoretical investigation of a disordered Stoner Wohlfarth model, as well as micromagnetic simulations. I show that the fluctuations in the total anisotropy and therefore in the switching fields are controlled by a single dimensionless parameter. The theoretical findings are well confirmed by micromagnetic simulations of many different samples.
\end{abstract}

PACS numbers: 85.75.B, 85.70.K, 75.75

Keywords: magnetic nanostructures, disorder, magnetic switching

\footnotetext{
*Corresponding author. Fax: +1 914945 4421; email: v2braun@us.ibm.com
} 


\section{INTRODUCTION}

With the development of integrated magneto-resistive memory devices the need to produce very many magnetic cells with reproducible magnetic switching behavior has arisen. As the dimensions of these devices reach orders of a few 100nm, the switching behavior is to good approximation that of a single domain particle, and mainly controlled by the total magnetic anisotropy. The magnetic anisotropy contains contributions from various sources, but for such small devices the contributions from the shape anisotropy and from the crystalline (i.e. material) anisotropy dominate. For a given magnetic material, the former can be controlled to a good degree by the aspect ratio and the thickness of the magnetic cells, while the latter depends on the actual microscopic structure. Typically magnetic films are polycrystalline with grain sizes of the order of a few nm to a few 10nm. The grains are oriented randomly. Since the preferred directions of the crystalline anisotropy are defined by the crystal axes, this leads to locally varying anisotropy contributions. Depending on the relative strength of shape anisotropy and crystalline anisotropy, one may therefore expect a more or less pronounced random component in the switching field. It is the purpose of this paper to assess quantitatively how much fluctuation one may expect depending on the various parameters in the problem.

A lot of work in material science has been done over many years to examine how magnetic parameters are influenced by the composition and micro structure of thin films ${ }^{1-9}$. Models of disordered ferromagnets were studied analytically by Ignatchenko and co-workers in the late 1970s and early 1980s (see ${ }^{10}$ and references therein) as well as by Kronmüller and coworkers ${ }^{12}$. They derived "laws of approach of the magnetization to saturation". Such laws were used as early as 1931 for the characterization of materials ${ }^{13}$. The laws derived by Ignatchenko et al. allowed to extract quantitative information about the correlations of local magnetic anisotropy ${ }^{11}$. The focus of these works was, however, on average macroscopic characteristics, like coercive fields or remanent magnetizations, and not on fluctuation of switching fields in small magnetic elements. Ref. ${ }^{14}$ deals with the latter problem by mi- 
cromagnetic simulations, but without a simple model presented the physical insight and predictive power of this analysis is limited. Also in the context of recording media, granular materials were extensively simulated ${ }^{15}$. Experimentally it is very difficult to distinguish the contributions of different origins to fluctuations in switching fields. In particular, fluctuations in the shapes of magnetic cells may easily mask fluctuations arising from the material.

In the following I will introduce a disordered Stoner Wohlfarth model, a simple model that allows to address the question of disorder induced fluctuations of the switching fields analytically. I will show that within this model the joint probability distribution $P(K, \gamma)$ for the total anisotropy strength $K$ and the overall preferred direction $\gamma$ is controlled by a single dimensionless parameter $\nu$. If we denote by $K_{1}$ the uniaxial shape anisotropy, by $K_{\mathrm{cr}}$ the crystalline (i.e. bulk single crystal) anisotropy, by $N$ the typical number of crystallites in the sample, and by $\alpha$ a number of order unity that depends on the shape of the crystallites, the parameter $\nu$ is given by

$$
\nu=\frac{N}{\alpha}\left(\frac{K_{1}}{K_{\mathrm{cr}}}\right)^{2} .
$$

The fluctuations of the switching field decay like $1 / \sqrt{\nu}$ for large $\nu$. All of this will be derived in Sec.II. Sec.III is devoted to numerical verification by micromagnetic modeling, and Sec.IV contains the conclusions.

\section{THE DISORDERED STONER WOHLFARTH MODEL}

Suppose the magnetic cell consists of $N$ crystallites, with respective volumes $V_{l}$, easy axis direction $\gamma_{l}(l=1, \ldots, N)$ and crystalline anisotropy $K_{\mathrm{cr}}$. For the moment let us consider the simplest case in which each crystallite gives rise to a uniaxial anisotropy. Further assume that the magnetization $M$ is uniform across the sample, with components uniquely specified by the angle $\theta, M_{x}=M_{s} \cos \theta, M_{y}=M_{s} \sin \theta$, where $M_{s}$ is the saturation magnetization. This approximation works well for sub- $\mu$ m sized magnetic cells, which are too small to hold domain walls. In an external magnetic field in the cell plane, $H=\left(H_{x}, H_{y}\right)$, the total energy density then reads 


$$
E / V=K_{1} \sin ^{2} \theta+K_{\mathrm{cr}} \sum_{l=1}^{N} v_{l} \sin ^{2}\left(\theta-\gamma_{l}\right)-H_{x} M_{\mathrm{s}} \cos \theta-H_{y} M_{\mathrm{s}} \sin \theta,
$$

where $v_{l}=V_{l} / V$ are the volume fractions of the crystallites ( $V$ denotes the total volume of the magnetic cell). Note that the forms of the crystallites do not enter at this point. The first and the last two terms on the right hand side correspond to the ordinary Stoner Wohlfarth model ${ }^{16}$.

In order to figure out the total anisotropy resulting from eq.(2) we need to understand how the different anisotropy terms add up. Let us start by looking at just two uniaxial anisotropies $K_{1}$ and $K_{2}$ with preferred angles $\gamma_{1}=0$ and $\gamma_{2}$. The energy density is then given by

$$
E(\theta) / V=K_{1} \sin ^{2} \theta+K_{2} \sin ^{2}\left(\theta-\gamma_{2}\right)
$$

We can rewrite this as

$$
E(\theta) / V=\epsilon+K \sin ^{2}(\theta-\gamma)
$$

where $\epsilon$ is a constant independent of $\theta$, and $K$ and $\gamma$ are the new anisotropy strength and preferred direction, respectively. By expanding the $\sin ^{2}$ terms in both eqs.(3) and (4) one easily convinces oneself that the three parameters $\epsilon, K$ and $\gamma$ are related to $K_{1}, K_{2}$ and $\gamma_{2}$ by the three equations

$$
\begin{aligned}
K_{1}+K_{2} \cos 2 \gamma_{2} & =K \cos 2 \gamma, \\
K_{2} \sin 2 \gamma_{2} & =K \sin 2 \gamma, \\
K_{2} \sin ^{2} \gamma_{2} & =K \sin ^{2} \gamma+\epsilon .
\end{aligned}
$$

I will use the convention that all anisotropy constants are positive $\left(0 \leq K_{1}, K_{2}, K \leq \infty\right)$ and all uniaxial preference angles are counted in the interval $-\pi / 2 \leq \gamma_{2}, \gamma<\pi / 2$. The system of equations is then solved uniquely for all $K_{1}, K_{2}$, and $\gamma_{2}$ by

$$
\begin{aligned}
\gamma & =\frac{1}{2} \operatorname{sign}\left(\gamma_{2}\right) \arccos \left(\frac{K_{1}+K_{2} \cos 2 \gamma_{2}}{K}\right), \\
K & =\sqrt{K_{1}^{2}+K_{2}^{2}+2 K_{1} K_{2} \cos 2 \gamma_{2}},
\end{aligned}
$$


and a third equation determining $\epsilon$, which is, however, irrelevant for the following. Note that the arccos function returns a value in the interval $0 \ldots \pi$, which by the prefactor $1 / 2$ is remapped to the interval $0 \ldots \pi / 2$. Since $\gamma_{1}$ was chosen as zero, the second angle determines the sign of the angle of the overall preferred direction. Fig.1 shows how the overall angle depends on $\gamma_{2}$ for various values of the ratio $r=K_{2} / K_{1}$.

\section{FIGURES}

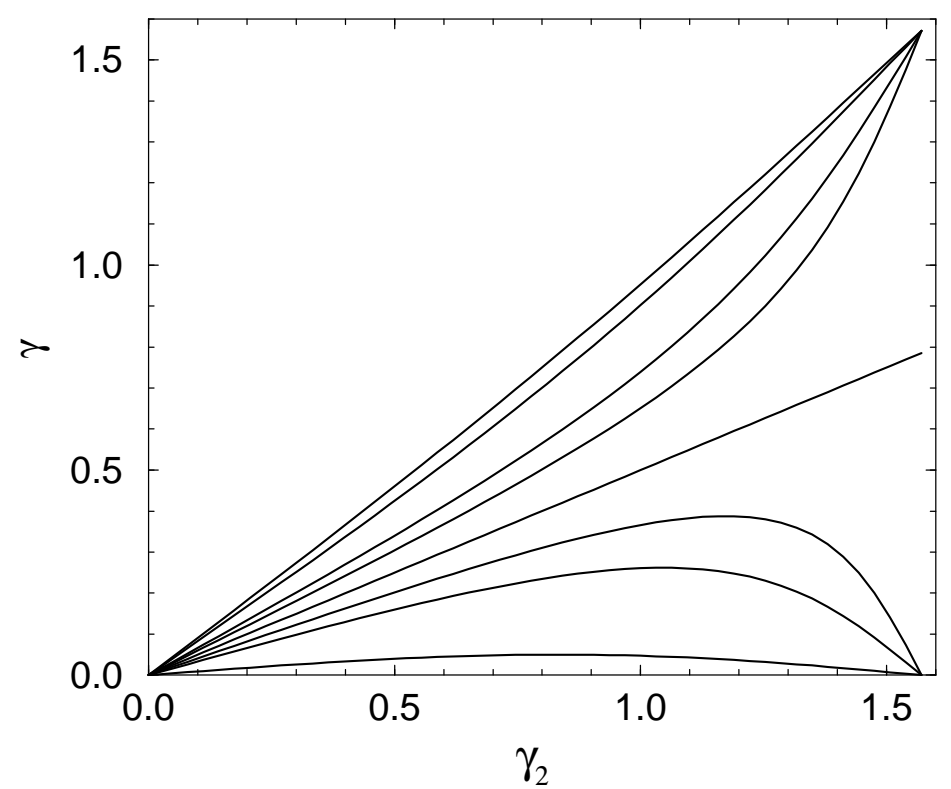

FIG. 1. Resulting angle from adding two uniaxial anisotropies (eq.8). For $r=K_{2} / K_{1}<1$, the maximum angle reached is smaller than $\pi / 4$. Only if $K_{2}$ dominates $(r>1)$, the angle $\pi / 2$ can be reached. The values of $r$ chosen are 0.1 (the curve with the smallest slope at $\gamma_{2}=0$ ), 0.5, 0.7, 1.0 (a straight line with slope 1/2), 1.5, 2.0, 5.0, and 10.0 (almost a straight line with slope 1.0). Only the first quadrant is shown, as $\gamma$ is an odd function of $\gamma_{2}$.

For $K_{2} / K_{1}<1$ the maximum angle reachable for the new overall preferred direction is smaller than $\pi / 4$ - the $K_{1}$ term always dominates and keeps the preferred direction close to zero. For $K_{1}=K_{2}$, an overall angle $\pi / 4$ can be reached at $\gamma_{2}=\pi / 2$, but eq.(9) shows that then the overall strength $K$ goes to zero: With two orthogonal preferred axes with anisotropy of equal strength, the total anisotropy vanishes indeed. For $K_{2}>K_{1}$, the overall 
preferred angle is dominated by the second anisotropy and therefore angles beyond $\pi / 4$ can be reached.

Concerning the total strength of the anisotropy, eq.(9) tells us that $K$ is obtained similarly as if two vectors with lengths and directions $K_{1}, K_{2}$ and directions $0, \gamma_{2}$ were added — with the only difference that the angle between the two vectors has to be replaced by twice its value in the rule for standard vector addition. Thus, the non-linear addition of uniaxial anisotropy terms is, for what concerns the resulting anisotropy strength, replaced by a linear vector addition, where the length of the vectors is given by the strengths of the anisotropy, and their enclosed angle is twice the angle between the original preferred directions.

Coming back to the disordered Stoner-Wohlfarth model, eq.(2), we now understand that the sum of anisotropy energies with random strength and preferred directions, i.e. the intrinsic anisotropy $K_{i}=K_{\mathrm{cr}} \sum_{l=1}^{N} v_{l} \sin ^{2}\left(\theta-\gamma_{l}\right)$, corresponds to a random walk in the plane: For the vector addition the relative angles are uniformly distributed in the interval $[-\pi, \pi[$, and the length of step $l$ is the random term $K_{\mathrm{cr}} v_{l}$. The unusual addition (8) of angles not withstanding, the resulting final orientation of the vector in the random walk is distributed uniformly over $\left[-\pi, \pi\left[\right.\right.$, for all $K_{i}$ since each single angle is. The random walk leads to a distribution of $K_{i}$ that is for $N \gg 1$ well approximated by a Gaussian centered at $K_{i}=0$ and with a variance $\sigma^{2}=\left\langle K_{i}^{2}\right\rangle=N K_{\mathrm{cr}}^{2}\left\langle v_{i}^{2}\right\rangle$. If we assume that the typical volume fraction is given by a typical crystallite dimension $a_{l}$ as $\left\langle v_{l}^{2}\right\rangle^{1 / 2}=\sqrt{\alpha}\left\langle a_{l}^{2}\right\rangle / L^{2}=\sqrt{\alpha} / N$, where $\alpha$ is a numerical prefactor depending on the distribution of crystallite areas (and thus on the shape of the crystallites) we obtain the scaling behavior $\sigma=\sqrt{\alpha} K_{\mathrm{cr}} / \sqrt{N}$, and the joint probability distribution $P\left(K_{i}, \gamma_{i}\right)$ of the intrinsic anisotropy and the preferred direction,

$$
P_{i}\left(K_{i}, \gamma_{i}\right)=\frac{\sqrt{2}}{\sqrt{\pi} \sigma} \exp \left(-\frac{K_{i}^{2}}{2 \sigma^{2}}\right) \frac{1}{\pi}
$$

for $-\pi / 2 \leq \gamma_{i}<\pi / 2$ and $0 \leq K_{i} \leq \infty$.

In order to determine the distribution of the total anisotropy, it is most convenient to start with an expression for the joint distribution of $K^{2}$ and the total preferred angle $\gamma$, employing once more the law for adding uniaxial anisotropies, eqs.(8) and (9). We have 


$$
\begin{aligned}
P\left(K^{2}, \gamma\right)= & \sqrt{\frac{2}{\pi}} \int_{0}^{\infty} \frac{d K_{i}}{\sigma} \int_{-\pi / 2}^{\pi / 2} \frac{d \gamma_{i}}{\pi} \delta\left(K_{i}^{2}+2 K_{1} K_{i} \cos 2 \gamma_{i}+K_{1}^{2}-K^{2}\right) \\
& \times \delta\left(\gamma-\frac{1}{2} \operatorname{sign}\left(\gamma_{i}\right) \arccos \left(\frac{K_{1}+K_{i} \cos 2 \gamma_{i}}{K}\right)\right) \exp \left(-K_{i}^{2} /\left(2 \sigma^{2}\right)\right)
\end{aligned}
$$

The two integrals are easily performed. We can then transform the distribution back to $P(K, \gamma)$, express $K$ with a dimensionless parameter $k$ as $K=k K_{1}$, and thus arrive at the final form

$$
P(K, \gamma)=\frac{1}{K_{1}} \sqrt{\frac{2 \nu}{\pi^{3}}} k \frac{\mathrm{e}^{-\left(k^{2}+1-2 k \cos 2 \gamma\right) \nu / 2}}{\sqrt{k^{2}+1-2 k \cos 2 \gamma}}
$$

with the dimensionless parameter $\nu$ defined previously in eq.(1). As is now obvious, the total distribution of anisotropies is uniquely specified by this parameter $\nu$, and so are all statistical properties of the switching fields.

The distribution is centered around $k=1$ and $\gamma=0$, and in fact diverges for these values for all parameters $\nu$. Typical values of $\nu$ for magnetic cells may vary over a large range. A rectangular 800x400x5 $\mathrm{nm}^{3}$ Permalloy cell leads to a shape anisotropy $K_{1}=44.4 \cdot 10^{3} \mathrm{erg} / \mathrm{cm}^{3}$. Assuming a crystalline anisotropy $K_{\mathrm{cr}}=1000 \mathrm{erg} / \mathrm{cm}^{3}$ and a typical crystallite size of 20nm, we have $\nu=1.58 \cdot 10^{6}$. Larger crystallites (say $\left\langle a_{i}^{2}\right\rangle^{1 / 2}=50 \mathrm{~nm}$ ), a smaller aspect ratio, and thinner films may reduce this value. A rectangular $500 \mathrm{x} 400 \mathrm{x} 3 \mathrm{~nm}^{3}$ cell has $\nu=10.2 \cdot 10^{3}$.

The distribution $P(K, \gamma)$ is most relevant for rotational remanence experiments on arrays of nominally identical magnetic cells ${ }^{17}$. In these experiments a strong magnetic field is applied at an angle $\beta$ relative to the nominal easy axis of the cells (as defined by shape anisotropy). Then the magnetic field is switched off, with the direction of the field preserved until zero field is reached, and one measures the remanent magnetization along the nominal easy axis as function of the angle $\beta$. In the strong magnetic fields (i.e. field values outside all astroids of the cells), a cell will always align to good approximation to the field. But when the field is switched off, cells that saw a positive field component along their actual easy axis will remain in a state magnetized along their positive actual easy axis, while those which saw a negative field component relative to their actual easy axis will fall into a state magnetized along their negative actual easy axis. Thus, all that matters is the distribution 
of preferred angles $\gamma$, but not the strength of the anisotropy. Let us call " $x$-axis" the nominal easy direction, $M_{c}$ the average total magnetic moment of a single cell along the $x$-axis at zero field, and $n$ the number of cells in the array. If all cells had $\gamma=0$ there would be a sharp jump of the total $M_{x}$ of the array at $\beta=\pi / 2$ from $M_{x}=n M_{c}$ to $M_{x}=-n M_{c}$, if $\beta$ is cranked up from zero to $\pi$. A finite width of the distribution of $\gamma$ is reflected directly in the width of the transition. Integrating out $K$ in (12) we find the distribution of the preferred angles alone. Fig.2 shows the result obtained from numerically integrating

$$
P(\gamma)=\int_{0}^{\infty} d K P(K, \gamma)
$$

Note the rather non-Gaussian profiles, in particular the pronounced cusps at zero angle, even for very small values of $\nu$ where the distribution is almost homogeneous over the entire angle interval $-\pi / 2 \ldots \pi / 2$.
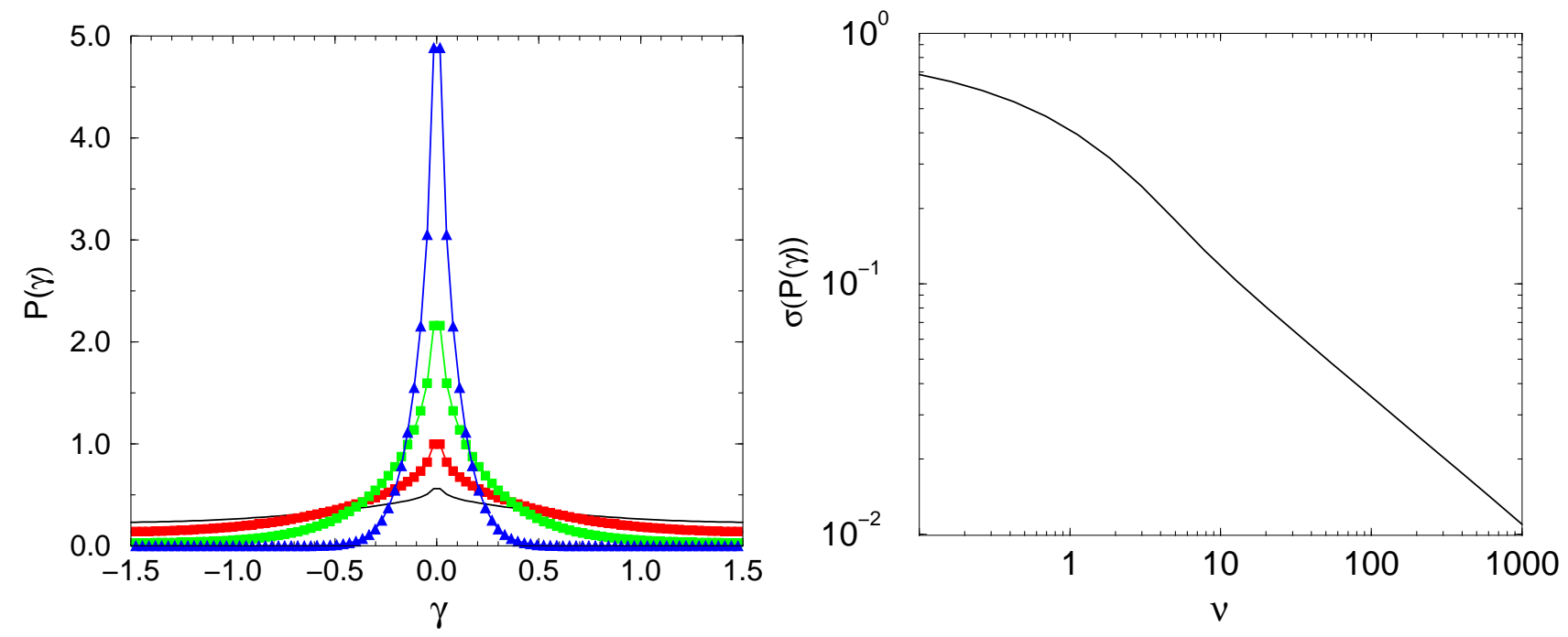

FIG. 2. Left: Distribution $P(\gamma)$ of the preferred angle, after integrating out the anisotropy strength $K$. The parameter $\nu$ ranges from $\nu=0.01$ (no symbols, flat curve) to $\nu=10$ (triangles). Right: Scaling of the width of the distribution $P(\gamma)$ with the parameter $\nu$.

The standard deviation of the distribution scales as $1 / \sqrt{\nu}$, as is shown in Fig.2. For comparison with actual experiments it is useful to note, however, that the cells in an array are typically not identical, even concerning their geometry. Fluctuations in the shape due to lithography errors and etch processes will lead to additional fluctuations in $K_{1}$ that can 
mask fluctuations due to the intrinsic material anisotropy.

\section{A. Switching field fluctuations}

Let us now have a look at the consequences for the distribution of switching fields. As is well known, the energy density (2) without the fluctuating term $\left(K_{\mathrm{cr}}=0\right)$ leads to a stability region in the $\left(H_{x}, H_{y}\right)$ plane given by the astroid $^{16}$

$$
\begin{aligned}
& h_{x, 0}(\theta) \equiv \frac{M_{s} H_{x}(\theta)}{2 K_{1}}=-\cos ^{3} \theta, \\
& h_{y, 0}(\theta) \equiv \frac{M_{s} H_{y}(\theta)}{2 K_{1}}=\sin ^{3} \theta .
\end{aligned}
$$

The two equations are obtained by setting simultaneously $\partial_{\theta} E(\theta) / V=0$ and $\partial_{\theta}^{2} E(\theta) / V=0$, which leads to the field combinations $\left(H_{x}(\theta), H_{y}(\theta)\right)$ where the nature of equilibrium points changes from stable to unstable (or from unstable to stable). A magnetic cell in a stable state with $M_{x}<0$ switches to a state $M_{x}>0$ if a field combination is applied that lies outside of the boundary (14), (15) in the positive half plane $h_{x}>0$ (and correspondingly for $\left.M_{x}<0\right)$.

Including the random crystalline contributions in eq.(2) we now have

$$
E(\theta) / V=K \sin ^{2}(\theta-\gamma)-M_{s} H_{x} \cos \theta-M_{s} H_{y} \sin \theta
$$

for the total energy density for a given cell (up to the irrelevant global shift $\epsilon$ ), where $K$ and $\gamma$ are distributed according to (12). Since the easy axis direction defines the orientation of the coordinate frame relative to which the Stoner-Wohlfarth astroid is measured, it is clear even without calculation that the energy density (16) leads for each $K$ and $\gamma$ again to an ideal astroid that is rotated by the angle $\gamma$ and changed in size by a factor $K / K_{1}$ (if magnetic fields are still measured in units of $2 K_{1} / M_{s}$, the normalization used in eqs. $\left.(14,15)\right)$. In the explicit form of the stability boundary derived again from $\partial_{\theta} E(\theta)=0=\partial_{\theta}^{2} E(\theta)$,

$$
\begin{aligned}
& h_{x}(\theta)=\frac{M_{s} H_{x}(\theta)}{2 K_{1}}=-\frac{K}{4 K_{1}}(\cos (3 \theta-2 \gamma)+3 \cos (\theta-2 \gamma)), \\
& h_{y}(\theta)=\frac{M_{s} H_{y}(\theta)}{2 K_{1}}=\frac{K}{4 K_{1}}(-\sin (3 \theta-2 \gamma)+3 \sin (\theta-2 \gamma)),
\end{aligned}
$$


the rotation of the astroid is somewhat obscured by the fact that $\theta$ is not the polar angle of the astroid, but just a parameter in a parametric representation. One easily convinces oneself, though, that acting on $\left(h_{x, 0}, h_{y, 0}\right)$ with a rotation matrix corresponding to an angle $\gamma$ and with an overall factor $K / K_{1}$ reproduces $(17),(18)$.

Eqs.(17) and (18) can be used together with (12) to calculate an average astroid as well as fluctuations around it. It turns out, however, that for this purpose it is more convenient to go back one step in the calculation and keep the total intrinsic anisotropy $K_{i}$ separate from the deterministic anisotropy $K_{1}$, since $K_{i}$ is simply Gaussian distributed, eq.(10). This will prove useful in Sec.II B as well, where we will look at the combination of random cubic crystalline anisotropy with uniaxial shape anisotropy. The latter situation will not even allow for defining an overall anisotropy with a single anisotropy constant. Let us therefore start from an energy density

$$
E(\theta) / V=K_{1} \sin ^{2} \theta+K_{i} \sin ^{2}\left(\theta-\gamma_{i}\right)-M_{s} H_{x} \cos \theta-M_{s} H_{y} \sin \theta
$$

with $K_{i}, \gamma_{i}$ distributed according to (10). If we express $K_{i}$ in units of $K_{1}, K_{i}=k_{i} K_{1}$, we arrive at

$$
\begin{aligned}
& h_{x}(\theta)=\frac{M_{s} H_{x}(\theta)}{2 K_{1}}=-\frac{k_{i}}{4}\left(\cos \left(3 \theta-2 \gamma_{i}\right)+3 \cos \left(\theta-2 \gamma_{i}\right)\right)-\cos ^{3} \theta, \\
& h_{y}(\theta)=\frac{M_{s} H_{y}(\theta)}{2 K_{1}}=-\frac{k_{i}}{4}\left(\sin \left(3 \theta-2 \gamma_{i}\right)-3 \sin \left(\theta-2 \gamma_{i}\right)\right)+\sin ^{3} \theta .
\end{aligned}
$$

Before presenting the results for the mean values and fluctuations of the switching fields, let me note that depending on the measurement performed different ways of averaging might be relevant. If we are interested in the fluctuation of the switching field for a given direction of the applied field and if the fluctuations are small, i.e. $\nu \gg 1$, the fluctuations for a fixed parameter $\theta$ are relevant. The parameter $\theta$ will be given to good approximation for all astroids by the unperturbed astroid (14), (15),

$$
\theta=\arctan \left(\frac{H_{y}}{H_{x}}\right)^{1 / 3} \text {. }
$$

However, when the fluctuations are larger, or if we are interested in swept astroids where $H_{y}$ is kept fixed and $H_{x}$ is swept, so that by definition all fluctuations are in $H_{x}$, the situation 
is much more complex. One will then have to calculate the relevant $\theta$ for each realization of the disorder separately. This will be discussed elsewhere ${ }^{20}$.

Here I will assume that the disorder induced fluctuations are small and that the point on the astroid where we are interested in the variation of the switching fields is sufficiently well described by $\theta$ as obtained from eq.(22). Since $k_{i}$ enters only linearly in (20), (21) and is distributed Gaussian, and since $\gamma_{i}$ is distributed uniformly over the interval $-\pi / 2 \ldots \pi / 2$, we find immediately that the average astroid is the ideal astroid given in eqs.(14),(15). The standard deviation of the switching field in units of $H_{c}=2 K_{1} / M_{s}$ is given by

$$
\sigma_{H x, H y}=\frac{H_{c}}{4} \frac{1}{\sqrt{\nu}} \sqrt{5 \pm 3 \cos 2 \theta},
$$

where $\sigma_{H x}$ comes with the positive sign under the square root, $\sigma_{H y}$ with the negative. Thus, the fluctuations in the switching field scale like $1 / \sqrt{\nu}$. For soft magnetic materials like permalloy and magnetic cells with aspect ratios not too close to unity, $K_{1}$ is dominated by shape anisotropy, which in turn is proportional to the aspect ratio over a wide range. We

conclude that the "array quality factor" $18 H_{c} / \sigma_{H x}$ for fixed cell width should be proportional to the aspect ratio of the cells to the power $3 / 2$.

\section{B. Cubic Crystalline Anisotropy}

A material with cubic anisotropy has an energy density that depends on the direction cosines $\alpha_{x}, \alpha_{y}$ and $\alpha_{z}$ of the magnetization with the crystal axes according to

$$
E / V=C\left(\alpha_{x}^{2} \alpha_{y}^{2}+\alpha_{x}^{2} \alpha_{z}^{2}+\alpha_{y}^{2} \alpha_{z}^{2}\right),
$$

where $C$ is the lowest order cubic anisotropy constant ${ }^{19}$. I will assume in the following that the crystallites all have a $z$-axis perpendicular to the film, i.e. the films are supposed to be well ordered in $z$-direction. This is a natural assumption for flat crystallites with lateral dimensions of a few $10 \mathrm{~nm}$ and films only a few $\mathrm{nm}$ thick, even though uniform distributions

of the crystal axis on cones have been observed in $50 \mathrm{~nm}$ thick films ${ }^{9}$. Furthermore, if the 
magnetization is effectively restricted to the plane of the film (for sufficiently thin films this is always the case), we have $\alpha_{z}=0$. I parameterize the magnetization in the plane again by an angle $\theta$ with respect to the $x$-axis. We then have $\alpha_{x}=\cos \theta, \alpha_{y}=\sin \theta$, and the expression reduces to

$$
E / V=\frac{C}{4} \sin ^{2} 2 \theta
$$

Cubic anisotropy projected to a (001) crystal plane thus leads to a four-fold (or bi-axial) symmetry in that plane. And instead of the four-fold jagged astroid for purely uniaxial materials, the stability curve is now eight-fold jagged. Note that the shape anisotropy of the cells is still uniaxial, though. The disordered Stoner Wohlfarth model for materials with the projected cubic crystalline anisotropy thus reads

$$
E / V=K_{1} \sin ^{2} \theta+\frac{C}{4} \sum_{i=1}^{N} v_{i} \sin ^{2}\left(2\left(\theta-\gamma_{i}\right)\right)-H_{x} M_{\mathrm{s}} \cos \theta-H_{y} M_{\mathrm{s}} \sin \theta,
$$

where I assume again that the orientations $\gamma_{i}$ of the crystallites are distributed uniformly over the entire relevant interval, i.e. $-\pi / 4 \leq \gamma_{i}<\pi / 4$. For adding only cubic anisotropies the same rules (8), (9) apply as were derived from adding uniaxial anisotropies. Indeed, in the derivation we can just replace $\theta \rightarrow 2 \theta$, and replace $K_{1}, K_{2}$ by two corresponding cubic anisotropy constants $C_{1}$ and $C_{2}$, and everything else goes through as before. The same is true for the random walk picture. Thus, many cubic anisotropy terms added up lead again to a cubic anisotropy with a distribution of the overall $C_{i}$ and $\gamma$ given by $2 P_{i}\left(C_{i}, \gamma\right)$, see eq. (10). The prefactor two is due to the fact that $\gamma$ now covers only half the previous interval. Things are different, however, when we combine the total cubic anisotropy with the uniaxial shape anisotropy. Obviously, the result will neither be a pure cubic anisotropy nor a pure uniaxial anisotropy, but rather a sum of two such terms. The boundary of the stability region derived from $\partial_{\theta} E(\theta)=0=\partial_{\theta}^{2} E(\theta)$, with

$$
E(\theta) / V=K_{1} \sin ^{2} \theta+\frac{C_{i}}{4} \sin ^{2}\left(2\left(\theta-\gamma_{i}\right)\right)-M_{s} H_{x} \cos \theta-M_{s} H_{y} \sin \theta
$$

now reads 


$$
\begin{aligned}
& h_{x}(\theta)=-\frac{M_{s} H_{x}(\theta)}{2 K_{1}}=\frac{c_{i}}{8}\left(5 \cos \left(3 \theta-4 \gamma_{i}\right)+3 \cos \left(5 \theta-4 \gamma_{i}\right)\right)-\cos ^{3} \theta \\
& h_{y}(\theta)=\frac{M_{s} H_{y}(\theta)}{2 K_{1}}=\frac{c_{i}}{8}\left(5 \sin \left(3 \theta-4 \gamma_{i}\right)-3 \sin \left(5 \theta-4 \gamma_{i}\right)\right)+\sin ^{3} \theta
\end{aligned}
$$

Depending on the relative strength $c_{i}=C_{i} / K_{1}$ and orientation of the total cubic intrinsic anisotropy, this boundary may be rather different from the ideal StonerWohlfarth astroid, as for example depicted in Fig.3. Depending on the parameters, little twists arise that might not always be resolvable in experiments, and give the impression of astroids broadened in one direction, or of kinks in the astroid sides.

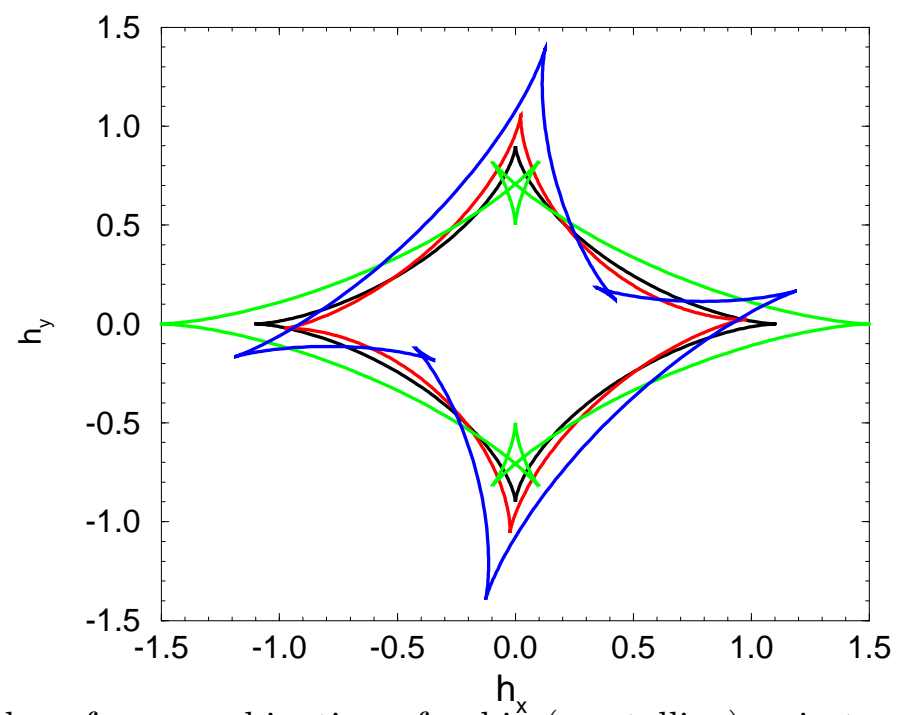

FIG. 3. Stability boundary for a combination of cubic (crystalline) anisotropy with uniaxial (shape) anisotropy. Moving from the curve with largest width in $y$-direction to the one with smallest width in $y$ direction, the curves correspond to the parameters 1.) $c_{i}=0.5, \gamma_{i}=0.5,2$.) $c_{i}=0.1, \gamma_{i}=0.5,3$.) $c_{i}=0.1, \gamma_{i}=0$, and 4.) $c_{i}=0.5, \gamma_{i}=0.0$, respectively.

As is evident from eqs.(28) and (29), the average astroid for fixed parameter $\theta$ is again the ideal astroid. The standard deviations of the switching fields are given by

$$
\sigma_{H x, H y}=\frac{H_{c}}{8} \frac{1}{\sqrt{\nu}} \sqrt{17 \pm 15 \cos 2 \theta}
$$

with the plus sign pertaining to $\sigma_{H x}$, and the minus sign to $\sigma_{H y}$. Note that the total uncertainty of the switching field $\sqrt{\sigma_{H x}^{2}+\sigma_{H y}^{2}}$ is independent of the parameter $\theta$ for both 
cubic and uniaxial crystalline anisotropy.

\section{NUMERICAL SIMULATIONS}

I performed micromagnetic simulations for many different cell types, varying cell size, aspect ratio, thickness, and material properties. A commercially available simulation package was used that allows to mimic a poly-crystalline structure with an adjustable average size of the crystallites. Each crystallite is then assigned a random preferred direction. Typically, 10 to 100 different disorder realizations were used for each type of cell simulated, and average value and standard deviation of the switching fields in easy direction (actually under a small angle of 4 degrees with respect to the easy axis in order to avoid the numerical problems related to catastrophic switching) were calculated. Fig.4 shows a cumulative plot for all samples with uniaxial or cubic crystalline anisotropy of the standard deviation of the switching field (in units of $K_{1}$ ) as function of the parameter $\nu{ }^{1}$ For the calculation of $\nu$, the numerically determined average value of $K_{1}$ was used, related to the switching field by $H_{c}=2 K_{1} / M_{s}$. For both types of crystalline anisotropy the decay of the fluctuations like $1 / \sqrt{\nu}$ is well observed over almost five orders of magnitude. For very large $\nu$ the fluctuations seem to decay slightly slower, but they might be limited by the finite field resolution, as well as the intrinsic fluctuations of the simulation program. Ideally one would expect all curves to collapse on a single one. The simulations show that the numerical constant $\alpha$ in the definition of $\nu$ does depend somewhat on the nominal sample properties.

\footnotetext{
${ }^{1}$ Note that a negative anisotropy constant corresponds to a preferred axis rotated by 90 degrees. As the crystallite axes are uniformly distributed over the full $\pi$ interval, $P(K, \gamma)$ should not depend on the sign of $K_{\mathrm{cr}}$. This was checked numerically by using some samples with negative $K_{\mathrm{cr}}$.
} 


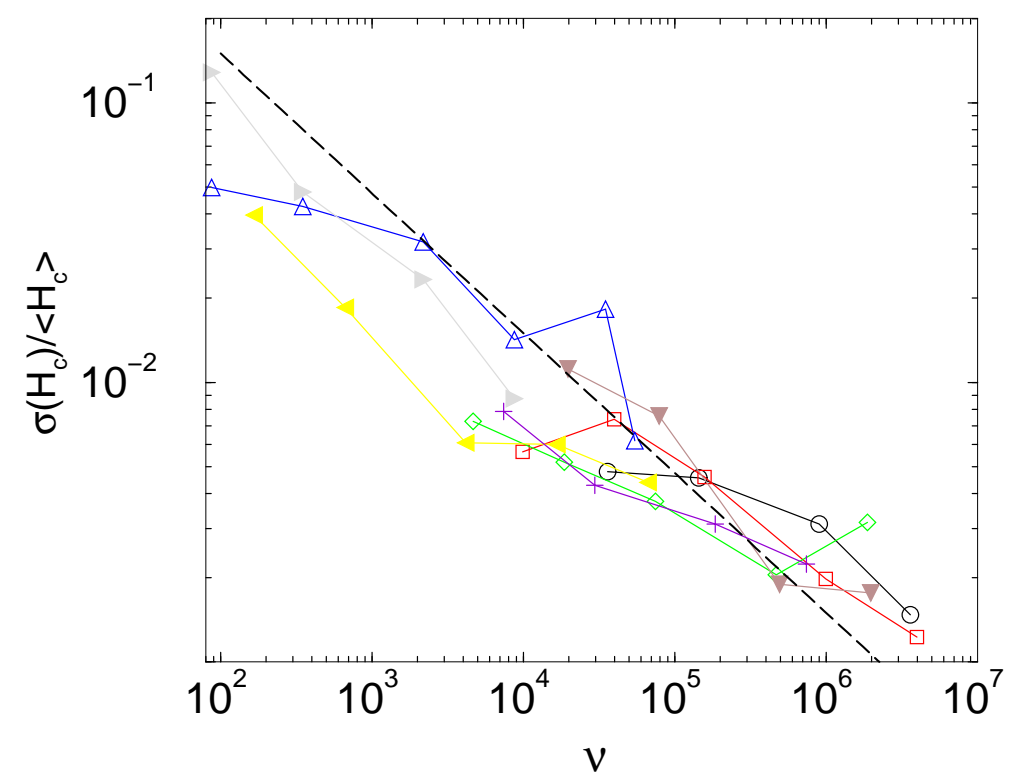

FIG. 4. Standard deviation of the switching field fluctuations in units of the average switching field for various systems. Empty circles: Ellipses $200 \times 400 \times 4 \mathrm{~nm}^{3}$ with $K_{\mathrm{cr}}=1.0 \cdot 10^{3} \mathrm{erg} / \mathrm{cm}^{3}$; empty squares: ellipses $300 \times 600 \times 4 \mathrm{~nm}^{3}, K_{\mathrm{cr}}=1.0 \cdot 10^{3} \mathrm{erg} / \mathrm{cm}^{3}$; empty diamonds: ellipses $300 \times 450 \times 4 \mathrm{~nm}^{3}$, $K_{\mathrm{cr}}=1.0 \cdot 10^{3} \mathrm{erg} / \mathrm{cm}^{3} ;$ empty triangles up: ellipses $200 \times 400 \times 4 \mathrm{~nm}^{3}, K_{\mathrm{cr}}=-15 \cdot 10^{3} \mathrm{erg} / \mathrm{cm}^{3} ;$ full triangles left: rectangles $200 \times 600 \times 4 \mathrm{~nm}^{3}$, uniaxial $K_{\mathrm{cr}}=-15 \cdot 10^{3} \mathrm{erg} / \mathrm{cm}^{3}$; full triangles down: $200 \times 400 \times 4 \mathrm{~nm}^{3}$, uniaxial $K_{\mathrm{cr}}=1.0 \cdot 10^{3} \mathrm{erg} / \mathrm{cm}^{3}$; full triangles right: ellipses $200 \times 400 \times 4 \mathrm{~nm}^{3}$, uniaxial $K_{\mathrm{cr}}=-15 \cdot 10^{3} \mathrm{erg} / \mathrm{cm}^{3}$; pluses: ellipses $300 \times 600 \times 4 \mathrm{~nm}^{3}$, uniaxial $K_{\mathrm{cr}}=1.0 \cdot 10^{3} \mathrm{erg} / \mathrm{cm}^{3}$. The lines are guides to the eye for data of the same nominal system, for which the crystallite size was varied, typically between 5 or $10 \mathrm{~nm}$ up to 100nm. The dashed straight line indicates the $1 / \sqrt{\nu}$ behavior.

\section{CONCLUSIONS}

I have presented a study of the influence of crystalline disorder on the switching behavior of small magnetic cells. Within a Stoner-Wohlfarth model with random anisotropy contributions I have derived the joint-probability distribution of the overall anisotropy strength and direction. The form of the distribution implies a dependence of the switching field fluctuations on a single parameter $\nu$, see eq.(1), in the form of a $1 / \sqrt{\nu}$ behavior. Also, the width of the transition in rotational remanence experiments should scale as $1 / \sqrt{\nu}$, and a 
broadening of the transition due to crystalline disorder should lead to a rather remarkable line shape. Micromagnetic simulations confirmed the scaling with $\nu$ both for uniaxial and cubic crystalline anisotropy.

Acknowledgements: It is my pleasure to thank David Abraham, Snorri Ingvarsson, Roger Koch, Yu Lu, John Slonczezwski, Solomon Woods and Philip Trouilloud for stimulating discussions. This work was done within the Magnetic RAM Developement Alliance (MDA) between Infineon Technologies AG and the IBM Corporation. 


\section{REFERENCES}

${ }^{1}$ C.L. Platt, A.E. Berkovitz, D.J. Smith, and M.R. McCartney, J. Appl. Phys. 88, 2058 (2000).

${ }^{2}$ Z.S. Shan, C.P. Luo, M. Azarisooreh, K. Honardoost, M. Russak, Y. Liu, J.P. Liu, and D.J. Sellmyer, IEEE Trans. Magn. 35, 2643 (1999).

${ }^{3}$ W. Weber, A. Bischof, R. Allenspach, C.H. Back, J. Fassbender, U. May, B. Schirmer, R.M. Jungblut, G. Güntherodt, B. Hillebrands, Phys. Rev. B 54, 4075 (1996).

${ }^{4}$ S.E. McKinlay, N. Fussing, R. Sinclair, and M. Doerner, IEEE Trans. Magn. 32, 3587 (1996).

${ }^{5}$ K.E. Johnson, M. Mirzamaani, and M.F. Doerner, IEEE Trans. Magn. 31, 2721 (1995).

${ }^{6}$ P.D. Kim, J.H. Song, I.A. Turpanov, S.V. Komogortsev, L.I. Semenov, M.M. Karpenko, and Y.H. Jeong, IEEE Trans. Magn. 35, 3097 (1999).

${ }^{7}$ L. Varga, H. Jiang, T.J. Klemmer, W.D. Doyle, and E.A. Payzant, J. Appl. Phys. 83, 5955 (1998).

${ }^{8}$ J.N. Zhou, J. Du, A.F. Khapikov, S. Wang, J.A. Barnard, J.W. Harrell, and W.D. Doyle, J. Appl. Phys., 87, 5624 (2000).

${ }^{9}$ P.W. Jang, D. Wang, and W.D. Doyle, J. Appl. Phys. 81, 4664 (1997).

${ }^{10}$ V.A. Ignatschenko, R.S. Iskhakov, and G.V. Popov, Zh. Eksp. Teor. Fiz 82, 1518 (1982) [Sov. Phys. JETP 55, 878 (1982)].

${ }^{11}$ V.A. Ignatschenko, R.S. Iskhakov, L.A. Chekanova, an d N.S. Chistyakov, Zh. Eksp. Teor. Fiz. 75, 653 (1978). [Sov. Phys. JETP 48, 328 (1978)].

${ }^{12}$ H. Kronmüller and J. Ulner, J. Magn. Mag. Mater. 8, 149 (1978).

${ }^{13}$ N.S. Akulov, Z. Phys. 69, 78 and 822 (1931). 
${ }^{14}$ Y. Zheng and J.-G. Zhu, J. Appl. Phys. 85, 4776 (1999).

${ }^{15}$ G. Hurley, T. Arnoldussen, R. Wood, D. Cheng, and M. Williams, IEEE Trans. Magn. 35, 2253 (1999).

${ }^{16}$ E.C. Stoner, E.P. Wohlfarth, Phil. Trans. Roy. Soc. A 240, 599 (1948).

${ }^{17}$ H.J. Richter and H. Hibst, J. Magn. Mag. Mater., 95, 118 (1991).

${ }^{18}$ D.W. Abraham, unpublished.

${ }^{19}$ A. Hubert and R. Schäfer, "Magnetic Domains", Springer-Verlag, Berlin, Heidelberg, New York (1998).

${ }^{20}$ D. Braun, unpublished. 